\title{
Patching and Suppression in Amblyopia: One Mechanism or Two?
}

\author{
Yiya Chen ${ }^{1 \dagger}$, Zhifen He ${ }^{1 \dagger}$, Yu Mao ${ }^{1 *}$, Hao Chen ${ }^{1 *}$, Jiawei Zhou ${ }^{1 *}$ and Robert F. Hess ${ }^{2}$ \\ ' School of Ophthalmology and Optometry and Eye Hospital, State Key Laboratory of Ophthalmology, Optometry and Vision \\ Science, Wenzhou Medical University, Wenzhou, China, ${ }^{2}$ McGill Vision Research, Department of Ophthalmology and Visual \\ Sciences, McGill University, Montreal, QC, Canada
}

Purpose: To determine if benefits from occlusion therapy are due to decreased suppression from the fellow eye in children with amblyopia.

Methods: Ten newly diagnosed amblyopes ( $7.2 \pm 1.4$ years old), two with strabismus and eight with anisometropia, participated. Patients were first given a 2-month period of refractive adaptation, followed by occlusion therapy (i.e., patching their fellow eye with an opaque patch for $4 \mathrm{~h}$ /day). Visual acuity of the amblyopic eye and interocular suppression were measured before and after 0.5, 1, 2, 4, and 6 months of occlusion

OPEN ACCESS

Edited by:

Gianluca Campana

University of Padua, Italy

Reviewed by:

Giulio Contemori, University of Padua, Italy

Marcello Maniglia,

The University of Alabama at Birmingham, United States

*Correspondence:

Yu Mao

maoyu_wmu@163.com Hao Chen

chenhao@mail.eye.ac.cn Jiawei Zhou

zhoujw@mail.eye.ac.cn

tThese authors have contributed equally to this work

Specialty section:

This article was submitted to Perception Science,

a section of the journal

Frontiers in Neuroscience

Received: 25 September 2019 Accepted: 03 December 2019 Published: 15 January 2020

Citation:

Chen Y, He Z, Mao Y, Chen H, Zhou J and Hess RF (2020) Patching and Suppression in Amblyopia: One

Mechanism or Two?

Front. Neurosci. 13:1364. doi: 10.3389/fnins.2019.01364 therapy. We quantified interocular suppression with a binocular phase combination task.

Results: Visual acuity (in logMAR) improved from $0.50 \pm 0.22$ (mean \pm SD) to $0.33 \pm 0.20$ for patients who finished a short-term (2 months) occlusion (A1-A10), from $0.53 \pm 0.20$ to $0.32 \pm 0.22$ for patients who finished a medium-term (4 months) occlusion (A1-A9), and from $0.48 \pm 0.19$ to $0.22 \pm 0.10$ for patients who finished a long-term (6 months) occlusion (A1-A8). Although their visual acuity significantly improved, their degree of suppression, which was abnormal in all cases, did not change consistently. This was true in all durations of occlusion therapy.

Conclusion: Reduced suppression from the fixing eye might not be result from occlusion therapy.

Keywords: patching, interocular suppression, amblyopia, binocular therapy, visual acuity

\section{INTRODUCTION}

For the past 250 years, occluding the fellow eye has been the standard therapy for amblyopia (de Buffon, 2005). Occlusion therapy "forces" the amblyopic eye to work. Emerging evidence suggests that $120-240 \mathrm{~h}$ of occlusion results in a 1-line improvement in visual acuity across all age groups when occlusion is effective (Stewart et al., 2007).

Recently, a new approach has been suggested. It involves re-establishing binocular vision as the first step by using binocularly based training therapy, which relies on a theory that amblyopia and abnormal interocular suppression are causally linked (Baker et al., 2008; Li et al., 2011; Birch, 2013; Ding et al., 2013; Zhou et al., 2013b,c, 2018). It improves not only binocular vision (Li et al., 2013; Hess and Thompson, 2015; Webber et al., 2016; Kelly et al., 2018) but also monocular acuity (Hess et al., 2010b, 2011; To et al., 2011; Li et al., 2013, 2014; Mansouri et al., 2014; Birch et al., 2015). Despite ongoing debate concerning which therapeutic approach is more effective (Holmes et al., 2016; Kelly et al., 2016; Gao et al., 2018; Manh et al., 2018; Pineles et al., 2019), one should bear in mind the possibility that the visual improvements from both approaches might involve the same mechanisms in the brain. If both approaches involve similar neural mechanisms, convenience and efficacy might determine which one to use. Otherwise, they could be used complementarily 
or specifically according to each patient's need. At first glance, both occlusion and binocular therapy might work to reduce suppression from the fellow eye which normally prevents the amblyopic eye from improving under normal binocular viewing conditions. Binocular therapy reduces suppression from the fellow eye under binocular viewing by reducing the amount of visual input to the fixing (suppressing) eye. Conversely, occlusion therapy might achieve a similar effect by entirely occluding the fixing (suppressing) eye. However, these two therapeutic protocols could involve disparate neural mechanisms as exemplified by their numerous differences. For example, they differ in the following ways: binocular outcomes [occlusion poor (Birch, 2013); binocular therapy - good (Hess et al., 2011; Knox et al., 2012; Li et al., 2013; Kelly et al., 2018)], age dependence [occlusion - only children (Epelbaum et al., 1993); binocular therapy - children and adults (Mansouri et al., 2014)], duration dependence [occlusion - 120-240 h (Stewart et al., 2007); binocular therapy - 20-40 h (Hess et al., 2010b; Mansouri et al., 2014)], and post-therapeutic remission [occlusion - 2427\% (Holmes et al., 2004; Bhola et al., 2006); binocular therapy no remission (Li et al., 2013, 2015; Birch et al., 2015)].

To assess whether occlusion therapy decreases interocular suppression in a similar fashion to binocular therapy (Hess et al., 2010a,b, 2011; Li et al., 2013; Spiegel et al., 2013), we measured interocular suppression at various time points (up to 6 months) in newly diagnosed patients. The patients had been prescribed $4 \mathrm{~h}$ per day of occlusion therapy after a 2 month period of refractive adaptation (Wang J. et al., 2018). We used a binocular phase combination task, a standard laboratory method (Ding and Sperling, 2006) to study binocular balance, e.g., amblyopia (Huang et al., 2009; Ding et al., 2013; Zhou et al., 2013b; Kwon et al., 2014) and strabismus (Ding et al., 2013; Feng et al., 2015; Zhou et al., 2017c), and binocular visual plasticity (Zhou et al., 2013a, 2014b, 2017a,b; Min et al., 2019; Sheynin et al., 2019), to quantify the degree of interocular suppression before and during the occlusion therapy. With this task, we were able to estimate the contribution of each eye to binocular vision at different interocular contrast ratios. We computed the interocular contrast ratio where two eyes contributed equally in binocular phase combination to quantify interocular suppression. We found that, although the visual acuity of patients' amblyopic eye was significantly improved by occlusion therapy, the degree of suppression did not significantly change even after 6 months of occlusion therapy. Therefore, our results suggest that the visual benefits provided by occlusion may not be due to reduced suppression from the fellow eye.

\section{MATERIALS AND METHODS}

\section{Participants}

Ten children with amblyopia $(\mathrm{A} 1-\mathrm{A} 10)$ with $(n=2)$ or without $(n=8)$ strabismus participated in our study. All patients were newly diagnosed and had no treatment history before participating in our study. Clinical details of patients before data collection are provided in Table 1. The definition of amblyopia for this study is conducted by PPP [American Academy of
Ophthalmology, Preferred Practice Patterns (Christiansen et al., 2018)]: patients had an interocular acuity difference of 2 lines (0.2 logMAR) or more, with an obvious cause (anisometropia, accommodation, strabismus, or deprivation). After 2 months of refractive adaptation (i.e., optical treatment), observers were asked to wear an opaque patch for $4 \mathrm{~h}$ each day. Except patient A9 who was followed up to 4 months and patient A10 who was followed only up to 2 months, all patients were followed up to 6 months. Individuals' visual acuity and interocular suppression were assessed before and after $0.5,1,2,4$, and 6 months of occlusion therapy.

\section{Apparatus}

The stimuli for interocular suppression measurement were generated and controlled by a PC computer running Matlab (MathWorks, Natick, MA, United States) with PsychToolBox 3.0.9 extension (Brainard, 1997; Pelli, 1997). The stimuli were presented on a gamma-corrected LG D2342PY 3D LED screen (LG Life Science, South Korea) with a $1920 \times 1080$ resolution, 8 bits of gray level, and a $60-\mathrm{Hz}$ refresh rate. Subjects viewed the display dichoptically with polarized glasses in a dimly lit room at a viewing distance of $136 \mathrm{~cm}$. The background luminance was $46.2 \mathrm{~cd} / \mathrm{m}^{2}$ on the screen and $18.8 \mathrm{~cd} / \mathrm{m}^{2}$ through the polarized glasses.

Participants' best spectacle-corrected visual acuity was measured monocularly using the Chinese Logarithmic Tumbling E Chart (Mou, 1966) at $5 \mathrm{~m}$. The visual acuity chart has 14 lines; the size of the optotypes changed from 1 to $-0.3 \operatorname{logMAR}$ in different lines with a step size of 0.1 log units. Patients were asked to report the orientation (the opening) of the letter " $E$ " one after another and were stopped when they could not respond within $10 \mathrm{~s}$. The amblyopic eye was always examined first during the experiment. Visual acuity was defined as the score associated with $75 \%$ correct judgments. This was achieved by measuring participants' percentage correct at different lines and using linear interpolation to calculate the score associated with 75\% correct judgments. Strabismus angle was measured using the prism cover test.

\section{Design}

A binocular phase combination paradigm (Ding and Sperling, 2006) was used to quantify the two eye's contribution to binocular percept (i.e., our measure of interocular suppression). In the test, two horizontal sine-wave gratings with equal and opposite phase shifts of $22.5^{\circ}$ (relative to the center of the screen) were dichoptically presented to the two eyes. The contrast was fixed as $100 \%$ in the amblyopic eye and varied with a ratio $\delta(\delta=[0$, $0.1,0.2,0.4,0.8,1])$ in the fellow eye. These contrast ratios were selected based on our previous studies (Huang et al., 2009; Zhou et al., 2013b) and recent papers in studying the effect of refractive adaptation in children and adults with amblyopia (Wang J. et al., 2018) as well as normative data (Wang Y. et al., 2018). The interocular suppression is quantified by the interocular contrast ratio (fellow eye/amblyopic eye) when the two eyes' information makes an equal contribution to binocular viewing, i.e., when the perceived phase of the binocularly combined grating is $0^{\circ}$ (Figure 1A). 
TABLE 1 | Baseline clinical details of the participants.

\begin{tabular}{|c|c|c|c|c|c|c|}
\hline Subject & Age/Sex & Cycloplegic refractive errors (OD/OS) & Amblyopia type & Squint (OD/OS) & Balance point & logMAR visual acuity (OD/OS) \\
\hline \multirow[t]{2}{*}{$\mathrm{A} 1 \triangle$} & $9 / \mathrm{M}$ & +3.75 & Aniso & $\varnothing$ & 0.37 & 0.27 \\
\hline & & Plano & & $\varnothing$ & & -0.03 \\
\hline \multirow[t]{2}{*}{ A2O } & $7 / \mathrm{M}$ & Plano & Aniso & $\varnothing$ & 0.26 & -0.03 \\
\hline & & $+2.00+0.50 \times 80$ & & $\varnothing$ & & 0.19 \\
\hline \multirow[t]{2}{*}{$\mathrm{A} 3 \nabla$} & $5 / F$ & $-1.00-1.00 \times 180$ & Aniso & $\varnothing$ & 0.43 & 0.27 \\
\hline & & $-6.00-2.00 \times 180$ & & $\varnothing$ & & 0.58 \\
\hline \multirow[t]{2}{*}{ A4 $\square$} & $6 / \mathrm{M}$ & +1.50 & Aniso & $\varnothing$ & 0.29 & -0.03 \\
\hline & & +5.00 & & $\varnothing$ & & 0.58 \\
\hline \multirow[t]{2}{*}{ A5॰ } & $8 / \mathrm{M}$ & Plano & Aniso & $\varnothing$ & 0.19 & -0.03 \\
\hline & & $+2.50+1.75 \times 80$ & & $\varnothing$ & & 0.58 \\
\hline \multirow[t]{2}{*}{$\mathrm{A} 6 \bullet$} & $6 / M$ & +3.50 & Accom + Stra & $\varnothing$ & 0.16 & 0.18 \\
\hline & & $+4.00+0.75 \times 95$ & & $\mathrm{ET} 5^{\circ}$ & & 0.67 \\
\hline \multirow[t]{2}{*}{ A7弥 } & $8 / F$ & +4.50 & Aniso & $\varnothing$ & 0.33 & 0.48 \\
\hline & & Plano & & $\varnothing$ & & -0.03 \\
\hline \multirow[t]{2}{*}{ A8D } & $6 / F$ & Plano & Aniso & $\varnothing$ & 0.23 & -0.03 \\
\hline & & $+2.00+1.75 \times 85$ & & $\varnothing$ & & 0.88 \\
\hline \multirow[t]{2}{*}{ A9 $\triangleleft$} & $9 / \mathrm{M}$ & +4.00 & Aniso & $\varnothing$ & 0.13 & 0.58 \\
\hline & & Plano & & $\varnothing$ & & -0.12 \\
\hline \multirow[t]{2}{*}{ A10匹 } & $8 / F$ & $+3.00+0.75 \times 90$ & Accom + Stra & $\varnothing$ & 0.09 & -0.03 \\
\hline & & $+3.50+1.00 \times 85$ & & $\mathrm{ET} 10^{\circ}$ & & 0.18 \\
\hline
\end{tabular}

Anis, anisometropia; Stra, strabismus; Accom, accommodation; ET, esotropia.

A

Phase $=-22.5^{\circ}$
Contrast $=\delta \cdot 100 \%$
Phase $=+22.5^{\circ}$
Contrast $=100 \%$

Contrast $=100 \%$
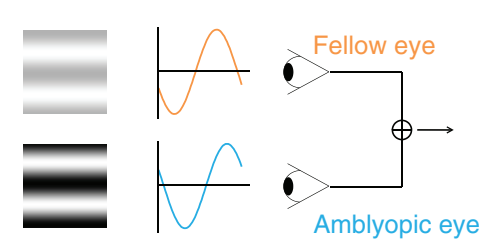

B

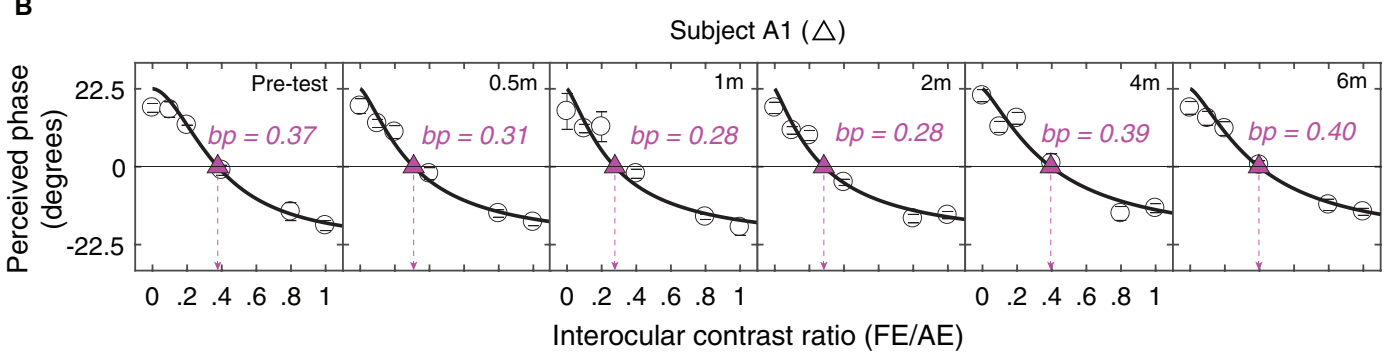

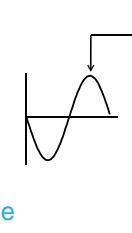

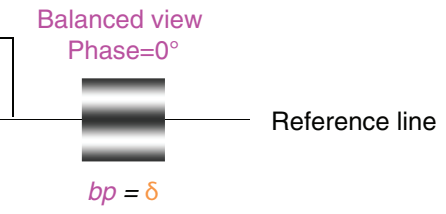

Amblyopic eye

$b p=\delta$ 
difference between the measured perceived phases in these two configurations. The two configurations at the six interocular contrast ratios were measured eight times using the method of constant stimuli. The perceived phase and its standard error were calculated based on these eight repetitions. There were in total 96 trials $(2$ configurations $\times 6$ interocular contrast ratios $\times 8$ repetitions) in one measure, which took about $20 \mathrm{~min}$ to finish. Voluntary breaks were allowed during the test. Practice trials were provided prior to data collection. The function of perceived phase vs. interocular contrast ratios (PvR function, Figure 1B) was then derived and was fitted by the attenuation gain control model (Huang et al., 2009) to get the effective contrast ratio at balance point (i.e., "bp") as illustrated in Figure 1. Some patients either found the baseline suppression measurements too difficult or were too variable in their responses; these patients were not enrolled in the study. Only patients who could complete the baseline measurements were enrolled in the study. All the patients who were enrolled in the study subsequently completed the study.

\section{Stimuli}

The stimulus configurations were identical to that previously described (Zhou et al., 2013b): two monocular horizontal sinewave gratings with different contrast but having equal and opposite phase shifts (relative to the center of the screen) were dichoptically presented in the middle of the two monocular displays. The sine-wave gratings had a period of two cycles, which subtended $2.0^{\circ}$ of visual angle (i.e., $1.00 \mathrm{cycle}^{\circ}$ ). A highcontrast frame $\left(0.11^{\circ}\right.$ in width and $6^{\circ}$ in length) with four white diagonal lines $\left(0.11^{\circ}\right.$ in width and $2.83^{\circ}$ in length $)$ was presented surrounding the grating in each eye to help observers maintain fusion. A 1-pixel black reference line was presented horizontally at the two sides of the gratings and observers were asked to move it to indicate the perceived phase after combination.

\section{Procedure}

An alignment task was provided at the beginning of each trial to make sure the two eyes' images were correctly fused. In the alignment task, a fixation marker was presented in the center of the larger high-contrast frame together with four white diagonal lines. This marker consisted of binocular fixation crosses $\left(100 \times 100\right.$ pixels $\left.^{2}\right)$ and four monocular dots (20 pixels diameter), two of which were in the first and third quadrants in the left eye and two of which were in the second and fourth quadrants in the right eye. Observers were instructed to move the image in their amblyopic eye using up, down, left, and right arrow keys to align the images from two eyes. After achieving stable fusion, observers were asked to press the "space" key. The corresponding coordinate between two eyes was then used in subsequent measurements. After that, a phase adjustment procedure (Zhou et al., 2014a) was used to measure the perceived phase of the binocularly combined gratings. Observers were asked to adjust the position of the reference line at the side of the grating to indicate the perceived phase of the cyclopean sine-wave grating, defined as the location of the center of the dark stripe of the grating. The reference line was presented with an initial position randomly ( -9 to 10 pixels) assigned relative to the center of the frame in each trial. It was moved with a fixed step size of 1 pixel, corresponding to $4^{\circ}$ phase angle of the sine-wave grating. During one trial, the gratings, frames, and reference lines were presented continually in the two eyes until subjects finished the phase adjustment. A typical trial lasted for about $10 \mathrm{~s}$.

\section{Curve Fits}

The PvR functions for different dichoptic pairs were fitted with the attenuation gain control model from Huang et al. (2009):

$$
\varphi=2 \tan ^{-1}\left[\frac{1-(\delta / b p)^{1+\gamma}}{1+(\delta / b p)^{1+\gamma}} \cdot \tan \left(\frac{\theta}{2}\right)\right]
$$

in which $\varphi$ is the measured perceived phase when the interocular signal contrast ratio is $\delta(\delta=[0,0.1,0.2,0.4,0.8,1.0]) ; \theta$ is the interocular phase difference (i.e., $45^{\circ}$ in our test) and the two free parameters, bp and $\gamma$, represent the effective contrast ratio at balance point (i.e., $\varphi=0^{\circ}$ ) and the non-linear factor in the binocular combination, respectively.

Curve fits were conducted in Matlab (MathWorks, Natick, MA, United States) using non-linear least squares method to minimized $\Sigma\left(\varphi_{\text {theory }}-\varphi_{\text {observed }}\right)^{2}$.

\section{Statistical Analysis}

All analyses were performed using IBM-SPSS 23.0 (IBM Inc., Armonk, NY, United States). Linear mixed-effects models were applied to explore associations of occlusion duration with visual acuity and interocular suppression. The relationship between changes of visual acuity and interocular suppression was assessed using Pearson correlation analysis.

\section{RESULTS}

\section{The Effect of Occlusion Therapy on Patients' Visual Acuity}

Figure 2 shows the visual acuity of the amblyopic eye as a function of occlusion durations in the 10 amblyopes (black symbols). The average results are also plotted in Figure 2 using open blue squares. Except patient A10 (घ), who showed almost no change of visual acuity, all other patients had clear improvement following the occlusion therapy.

For patients who finished the short-term occlusion therapy (i.e., 2 months; A1-A10), the average visual acuity of the amblyopic eye changed from $0.50 \pm 0.22$ (mean \pm SD) to $0.33 \pm 0.20$. For patients who finished the mediumterm occlusion therapy (i.e., 4 months; A1-A9), the average visual acuity of the amblyopic eye changed from $0.53 \pm 0.20$ (mean $\pm \mathrm{SD}$ ) to $0.32 \pm 0.22$. For patients who finished the long-term occlusion therapy (i.e., 6 months; A1-A8), the average visual acuity of the amblyopic eye changed from $0.48 \pm 0.19$ (mean $\pm \mathrm{SD}$ ) to $0.22 \pm 0.10$.

A linear mixed-effects model showed that the visual acuity of the amblyopic eye was significantly associated with occlusion durations with the estimated fixed effect being -0.031 


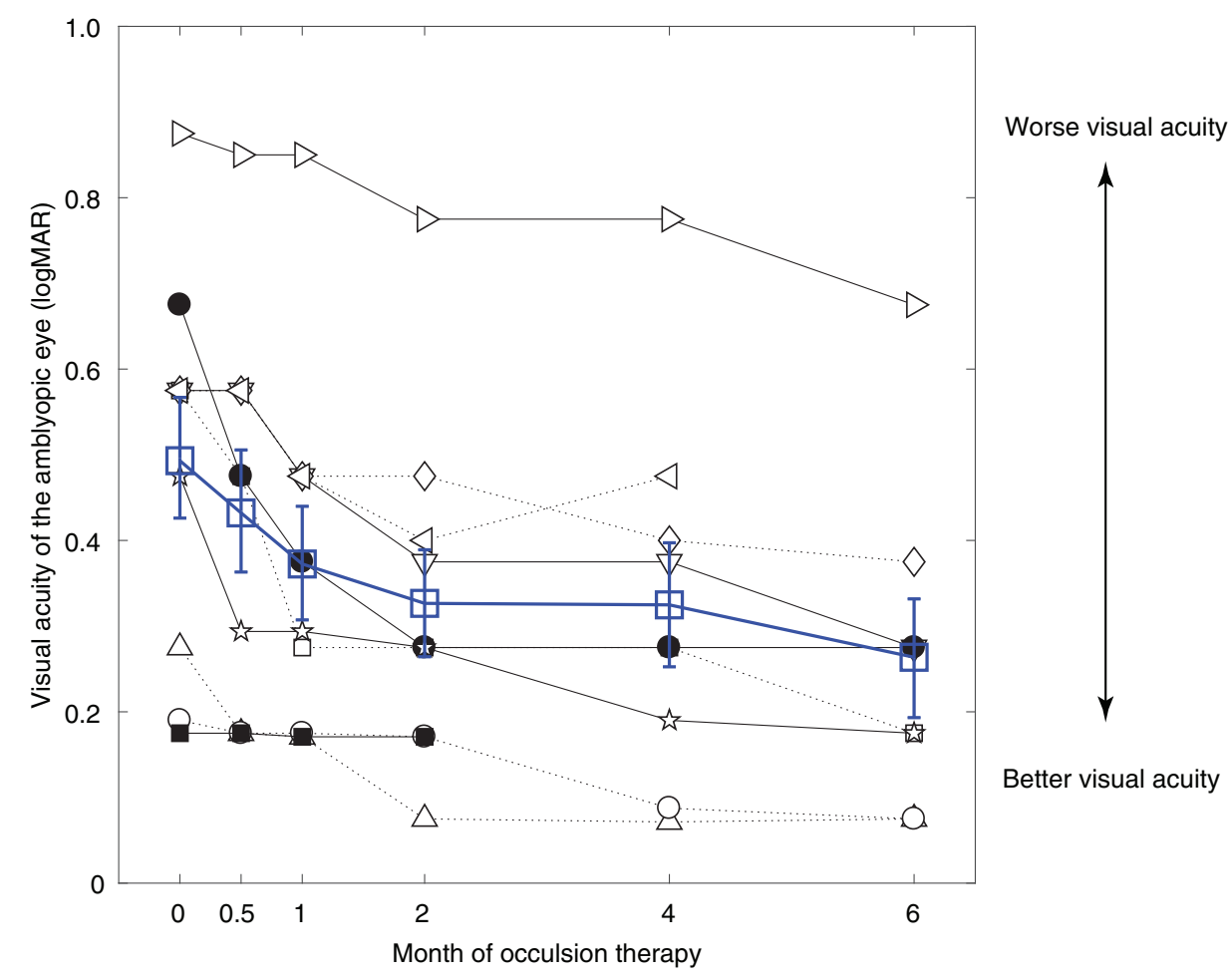

FIGURE 2 | Significant benefits on amblyopic eye's visual acuity from occlusion therapy. Individuals' visual acuity of the amblyopic eye as a function of the occlusion duration. Results of different patients were plotted using black symbols, and their average was plotted using blue open squares. Error bars represent SEM.

$(p<0.001)$. This indicated that the visual acuity of the amblyopic eye improved approximately $0.031 \operatorname{logMAR}$ per month during 6 months of occlusion therapy. On the other hand, the visual acuity of the fellow eye was not significantly changed over time during occlusion therapy $(p=0.169)$. These results suggest that the visual acuity benefits of the amblyopic eye could not be simply accounted for by a practice effect of repeated testing.

\section{The Effect of Occlusion Therapy on Patients' Interocular Suppression}

Patients were well-practiced before beginning the interocular suppression measurements (i.e., the binocular phase combination test). The quality of the data was excellent as the attenuation gain control model from Huang et al. (2009) fitted well to our data. The averaged goodness of fit for the patients were $0.90 \pm 0.14$ (pre-occlusion treatment; mean $\pm \mathrm{SD}$ ), $0.94 \pm 0.04$ (after 0.5 months of occlusion treatment), $0.96 \pm 0.03$ (after 1 month of occlusion treatment), $0.93 \pm 0.08$ (after 2 months of occlusion treatment), $0.95 \pm 0.03$ (after 4 months of occlusion treatment), and $0.95 \pm 0.05$ (after 6 months of occlusion treatment) at different time sessions. Figure 3 shows the interocular suppression (i.e., effective contrast ratio at balance point) as a function of occlusion treatment durations in the 10 amblyopes (black symbols). The average results were also plotted in Figure 3 using open blue squares. Except for patient A10 (-), who showed a dramatic increase of the balance point, all other patients had consistent and similar balance points even after
6 months of occlusion therapy. No patient reached the normal range derived from previous reports in normal adults, i.e., 0.93 (Wang Y. et al., 2018) or the ideal observer's level (i.e., 1.0).

For patients who finished the short-term occlusion therapy (i.e., 2 months; $\mathrm{A} 1-\mathrm{A} 10)$, the average effective contrast ratio at balance point changed from $0.25 \pm 0.11$ (mean \pm SD) to $0.27 \pm 0.11$. For patients who finished the medium-term occlusion therapy (i.e., 4 months; A1-A9), the average effective contrast ratio at balance point changed from $0.26 \pm 0.10$ (mean $\pm \mathrm{SD}$ ) to $0.27 \pm 0.10$. For patients who finished the long-term occlusion therapy (i.e., 6 months; A1-A8), the average effective contrast ratio at balance point changed from $0.28 \pm 0.09$ (mean $\pm \mathrm{SD}$ ) to $0.28 \pm 0.13$. A linear mixed-effects model showed that the effective contrast ratio at balance point was not significantly associated with occlusion durations $(p=0.309)$, which indicated no change of interocular suppression during occlusion therapy.

To better illustrate the change of effective contrast ratio at balance point, post-treatment balance points relative to the pretreatment balance points are plotted in Figure 4 for each patient at different occlusion stages (i.e., $0.5,1,2,4$ and 6 months). It is clear that most patients had a constant and consistent balance point (i.e., close to the identity line) after up to 6 months of occlusion therapy. Based on the effect size (i.e., change of balance point) and the variance in our samples at different occlusion durations, we found that the sample size would have to be at least 545, 2065, and 2742, respectively for 2, 4, and 6 months of occlusion treatment to reach an $80 \%$ power and two-tailed 


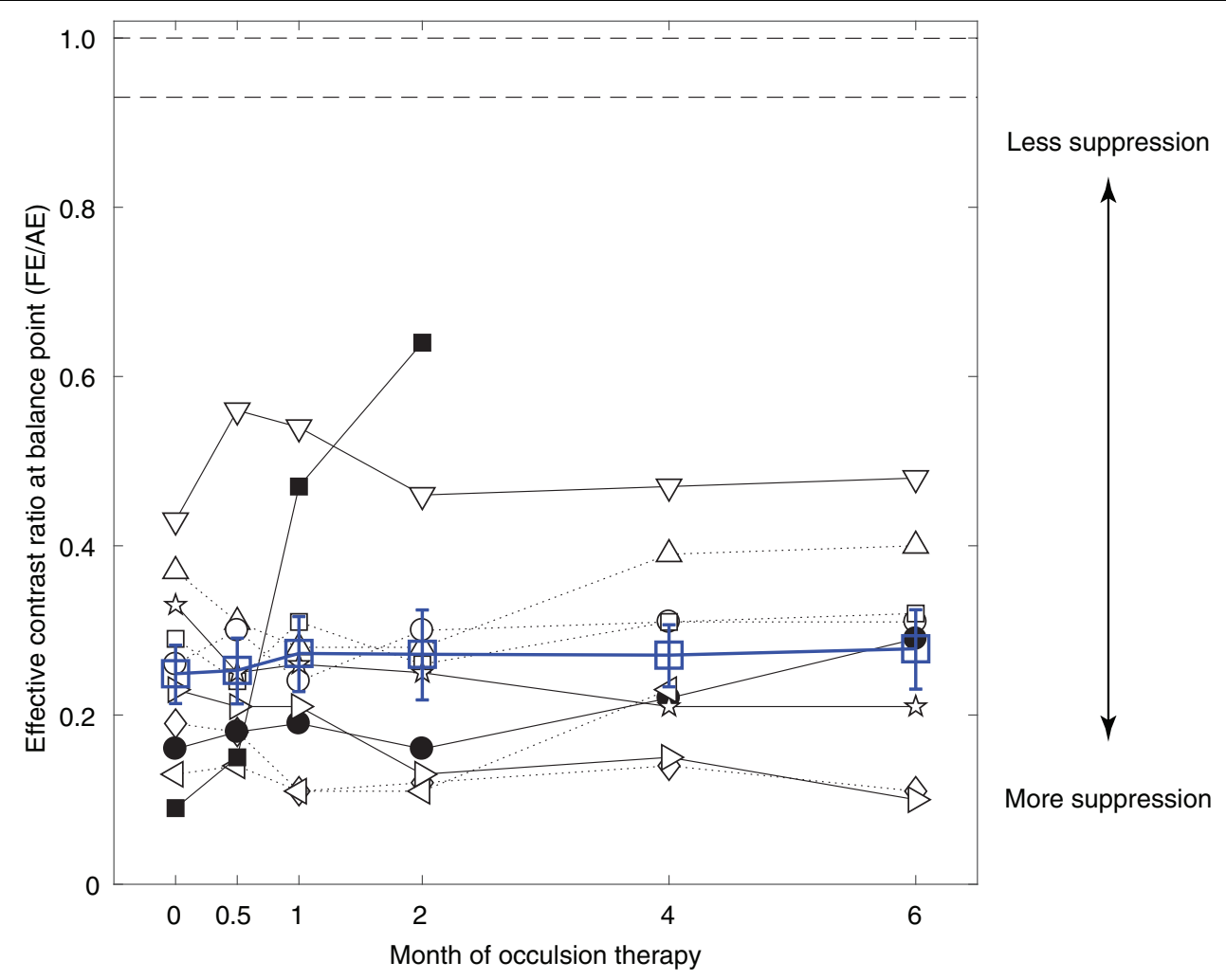

FIGURE 3 | No significant benefits on interocular suppression from occlusion therapy. Individuals' effective contrast ratios at balance point as a function of the occlusion duration. Results of different patients were plotted using black symbols, and their average was plotted using blue open squares. The two dashed lines at the top indicate the normal range of balance point derived from the literature, which is 0.93 from Wang Y. et al. (2018) (averaged from 144 adults) and 1.0 for ideal observer. Error bars represent SEM.

significance level at $\alpha=0.05$. These results in turn indicate that the change in suppression was not clinically meaningful. This demonstrates that these patients had significant suppression that did not change over the course of the treatment. This can be contrasted with the small but significant change (the average binocular gain was 0.11 in terms of the effective contrast ratio) in suppression that occurs as the result of a similar period of inverse occlusion in a similar age group using the same suppression measuring task (Zhou et al., 2019).

\section{The Relationship Between Changes of Visual Acuity and Interocular Suppression}

To better illustrate the relationship between the change of effective contrast ratio at balance point (our measure of suppression) and the change of amblyopic eye's visual acuity, we plotted post-treatment balance point change relative to the posttreatment visual acuity change in Figure 5 for each patient at different occlusion stages (i.e., $0.5,1,2,4$ and 6 months). It is clear that most patients had a constant and consistent balance point (i.e., points close to the horizontal line) and increased visual acuity (i.e., points shift to the left side from the vertical line) after up to 6 months of occlusion therapy. The two visual outcomes were not significantly correlated at any occlusion durations (for all, $p>0.10$, Pearson correlation analysis). This demonstrates that the change of balance point did not vary with the change of visual acuity.

\section{DISCUSSION}

Occlusion treatment - be it short-term, intermediate-term, or long-term - has been shown to improve visual acuity in about $50 \%$ of patients with amblyopia (Holmes et al., 2003, 2005; Repka et al., 2003). Our findings are in line with previous studies regarding the improvement in visual acuity from occlusion therapy. Despite the apparent improvement in visual acuity, the degree of interocular suppression, which was abnormal in all cases, did not change significantly. This was true for all durations of the occlusion treatment regime. However, the findings of Kelly et al. (2016) are in contrast with our results. They show that occlusion treatment for 2 weeks during which the fellow eye was deprived for $2 \mathrm{~h}$ per day reduces interocular suppression and facilitates recovery in binocular vision. We do not have any explanations for this change in suppression after a short period of occlusion therapy because our findings show no such change in the short or long terms.

We recruited 10 children with amblyopia (two with strabismus and eight without strabismus) in this study. 


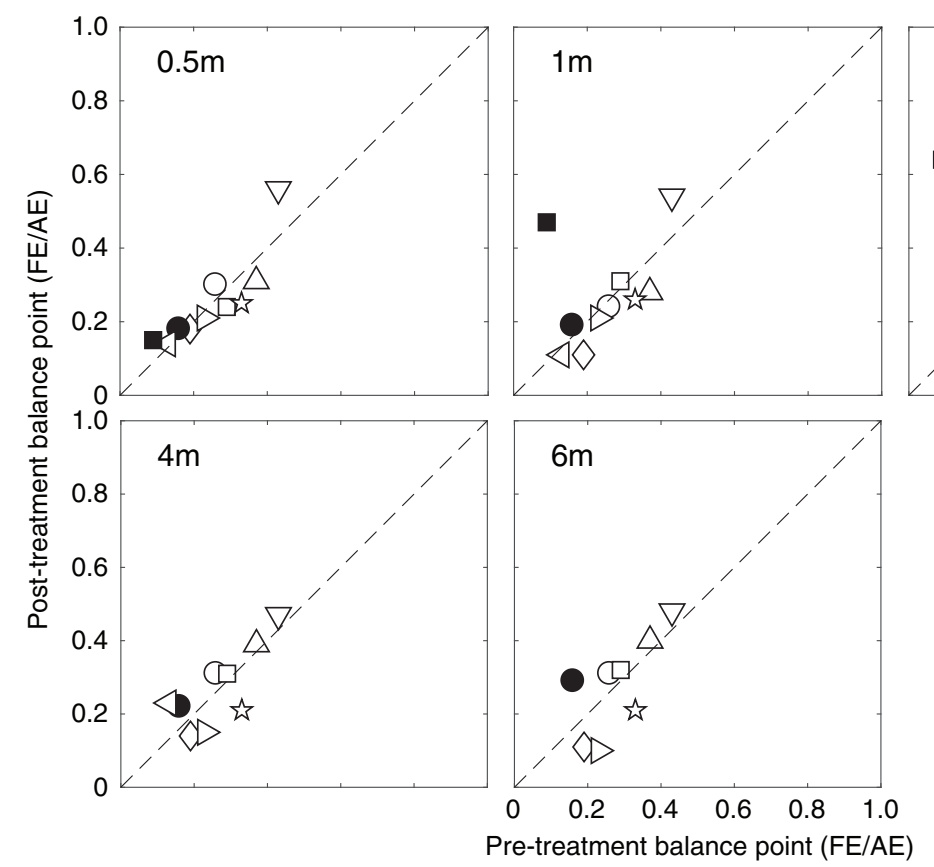

FIGURE 4 | Individuals' post-treatment balance points as a function of the pre-treatment balance points. Symbols above the dashed line (identity line) indicate less suppression after occlusion, while those below the dashed line indicate more suppression after occlusion.

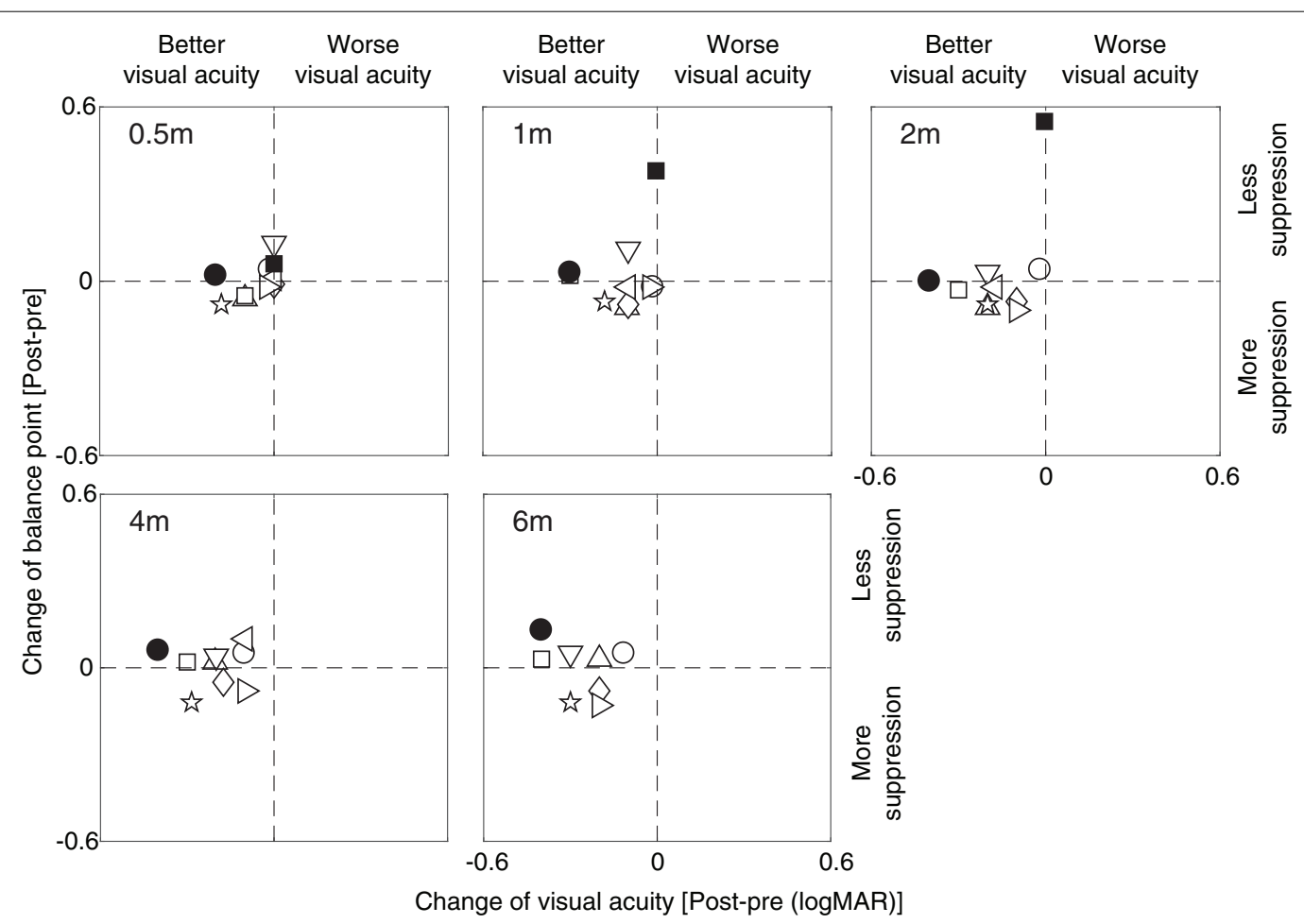

FIGURE 5 | The covariation between balance point change and visual acuity change. Symbols above the horizontal dashed line indicate less suppression after occlusion, while symbols below the horizontal dashed line indicate more suppression after occlusion; symbols at the left side of the vertical dashed line indicate better visual acuity after occlusion, while symbols at the right side of the vertical dashed line indicate worse visual acuity after occlusion. 
Although this sample size might appear to be too small to show a robust result, it had been chosen based on previous studies (Moseley et al., 1997; Pediatric Eye Disease Investigator Group Writing Committee Rutstein et al., 2010; Kehrein et al., 2016; Wang et al., 2016; Zhou et al., 2019). For example, the minimum sample size of six patients is adequate to achieve a power greater than $80 \%$ in detecting a visual acuity gain of $0.15 \pm 0.12 \log$ MAR (data from Wang et al., 2016). Similarly, the minimum sample size of two patients is adequate to achieve a power greater than $80 \%$ if the expected binocular gain in terms of the effective contrast ratio is $0.11 \pm 0.055$ (data from Zhou et al., 2019). The previous studies collectively reinforce the notion that our sample size of 10 patients with amblyopia is adequate to achieve a sufficient power to detect small changes in visual acuity and interocular suppression following occlusion therapy.

However, the visual acuity benefits did not show a significant correlation with reduced suppression in our patients. This finding is consistent with a previous study by Kehrein et al. (2016). They used a qualitative clinical test involving a red filter ladder (i.e., the Sbisa bar test) to quantify interocular suppression. They found no statistically significant change in interocular suppression after 4 months ( $6 \mathrm{~h}$ per day) of occlusion therapy. Their further analysis shows that the change of interocular suppression may be different between the subtypes of amblyopia: interocular suppression slightly declined after 4 months of occlusion treatment in amblyopes without strabismus (7/15), whereas that of amblyopes with strabismus did not (8/15). However, we did not find robust changes of interocular suppression in our anisometropic amblyopes (8/10). Since both studies contain a small number of subjects, whether this is due to the individual variability or a poor test-retest reliability of the quantitative clinical test, even in adults (Piano and Newsham, 2015), remains to be resolved. Furthermore, a luminance-based interocular suppression test (i.e., the Sbisa bar test) in the study of Kehrein et al. (2016) and a more quantitative laboratory, contrastbased interocular suppression test (i.e., the binocular phase combination task) in the current study might reflect different mechanisms that underlie interocular suppression (Chen et al., 2019). Nevertheless, both studies suggest that the visual acuity benefits from occlusion therapy are not correlated with those of reduced interocular suppression. In this respect, it is likely that the benefits from occlusion therapy could be from a different source than those of binocular treatment, which reduces suppressive effects from the fellow eye (Hess et al., 2011; Li et al., 2013; Spiegel et al., 2013). In fact, Mansouri et al. (2008) discovered the treatment effects serendipitously by measuring interocular suppression. They realized over several sessions of measurement that not only the degree of interocular suppression reduced (Mansouri et al., 2008) but also the visual acuity of the amblyopic eye improved (Hess et al., 2010a,b). Subsequent studies have confirmed this relationship (Hess et al., 2011; Li et al., 2013; Spiegel et al., 2013). This is clear in adults from the aforementioned studies but not so in children (although see Kelly et al., 2018), from whom it is more difficult to obtain valid measurements of interocular suppression.

The notion that monocular occlusion therapy and binocular therapy might involve different neural mechanisms is in line with several other notable differences between the effects of these two therapeutic approaches. First, occlusion is only effective in children up to the age of 17 , but it is ineffective in adults (Epelbaum et al., 1993). Binocular training has been shown to be effective in adults (Hess et al., 2010a,b, 2011; To et al., 2011; Li et al., 2013; Spiegel et al., 2013) and children (Knox et al., 2012; Li et al., 2014; Birch et al., 2015) with similar effectiveness. Second, a better binocular outcome has been achieved through binocular training than occlusion (Knox et al., 2012). Third, the treatment duration is of the order of 20-40 h for binocular training and over $120 \mathrm{~h}$ for occlusion (Stewart et al., 2004; Hess et al., 2010b). Fourth, the recurrence rate is high with occlusion [24-27\% (Holmes et al., 2004; Bhola et al., 2006)] and low with binocular training (Birch et al., 2015). Fifth, studies on children using the binocular approach have primarily examined children who failed to improve from occlusion therapy or who reached their best recovery after occlusion therapy (Knox et al., 2012; Li et al., 2014; Birch et al., 2015). Yet, the binocular approach has been shown to achieve additional benefit in visual acuity (Knox et al., 2012; Li et al., 2014; Birch et al., 2015).

Binocular training is based on the idea that reducing the contrast response from the fixing eye will result in less suppression of information in the neural circuits associated with the amblyopic eye (Zhou et al., 2018). This in turn will result in a more balanced interocular inhibition and subsequently enable information from the amblyopic eye to contribute more in binocular vision. Animal models suggest that GABA mediates this form of suppression in the primary visual cortex (Sengpiel et al., 1994, 2006) either via the longrange cortical horizontal fibers connecting large basket cells (Sengpiel et al., 1994, 2006) in superficial cortical layers that connect same and opposite eye domains (Buzas et al., 2001) or via binocular suppression by inhibitory interneurons receiving input from thalamocortical inputs and simple cells, occurring at the thalamo-cortical synapse. If the effects of occlusion do not rely on reduced interocular suppression, what could their neural basis be? One possibility is that it could be due to metaplasticity (Abraham and Bear, 1996). Meta-plasticity involves homeostatic regulation of synaptic plasticity. In the mouse, monocular deprivation leads to not only synaptic depression of deprived eye synapses but also potentiation of non-deprived eye synapses that is experience-dependent (Frenkel and Bear, 2004; Iny et al., 2006). Thus, by abolishing or severely reducing responses from the fixing eye with an occluder, improved correlation can be obtained between the previously weak synaptic responses and the post-synaptic activity of neurons. The only problem with this explanation is that the predicted response deficit to the occluded eye is rarely observed in the age range where occlusion is used.

If it is the case that these two therapeutic approaches have different sites of action, it raises the question that if they were used together, would their combined effects be mutually 
constructive or destructive. One suspects that the answer to this question might depend on the outcome measure. If the outcome measure was visual acuity, the two approaches may well constructively combine. However, if the outcome measure was binocular vision (including stereopsis), one might expect a destructive interference; monocular occlusion and binocular combination are, by definition, polar opposite procedures. Two different types of neural recovery in turn suggest two different components to the original neural deficit, one with a binocular basis and another with a monocular basis.

\section{Relevance to Other Monocular Interventions in Amblyopia}

Besides occlusion therapy and binocular training that we have mentioned above, there are several other monocular interventions to improve visual acuity in patients with amblyopia. One example of monocular intervention for treating amblyopia is a non-invasive brain stimulation technique. Thompson et al. (2008) firstly showed that transcranial magnetic stimulation (TMS) could temporarily improve the contrast sensitivity of the adult amblyopic eye. Later, by using daily continuous theta burst stimulation (cTBS) of the visual cortex, Clavagnier et al. (2013) found that the effect of daily cTBS on contrast sensitivity could be accumulated and is long lasting. The findings were also reported by other studies (Ding et al., 2016; Bocci et al., 2018). This is suggested to be a result of altering the balance between excitation and inhibition of targeted brain areas by the non-invasive brain stimulation (Nitsche and Paulus, 2000; Campana et al., 2016). This could well represent a more direct way of redressing the interocular imbalance that is due to suppression; this is yet to be determined. Another example of monocular intervention in amblyopia is monocular visual perceptual learning (PL). It relies on intensively visual training with specially designed visual tasks. There has been evidence that visual PL could improve an amblyopic eye's Vernier acuity (Levi et al., 1997), visual acuity (Polat et al., 2004), contrast sensitivity (Zhou et al., 2006), and other vision functions (Li and Levi, 2004; Levi, 2005; Huang et al., 2008; Astle et al., 2011). There is evidence that PL can enhance modulation in neuronal tuning in V1 to the trained stimulus and that this consequently facilitates visual functions (Huang et al., 2008; Zhou et al., 2012; Ren et al., 2016; Yu et al., 2016). The role of interocular suppression is less clear for this approach. Two recent studies have tried to answer this question but have come to opposing conclusions: Chen et al. (2016) found that monocular PL improved binocular combination in adult amblyopes. However, by using a similar training protocol, Jia et al. (2018), failed to find any significant effect of monocular PL on binocular phase combination. Considering that the training in these two studies was conducted at a high spatial frequency (cutoff spatial frequency), which was not matched with the spatial frequency of their interocular suppression measurements $(0.3$ or $1 \mathrm{c} /{ }^{\circ}$ ), it would be necessary for further studies to explore the effect of PL on binocular visual functions using an approach (Kwon et al., 2015; Birch et al., 2016; Wang et al., 2019) that would allow the effect of spatial frequency to be considered for suppression.

Unlike occlusion treatment, both non-invasive brain stimulation and monocular PL are effective in older adults as well and to act on a short time frame, closer to binocular therapies than to occlusion. Furthermore, the effects of daily cTBS and visual PL on amblyopia are long lasting ( $\mathrm{Li}$ and Levi, 2004; Levi and Li, 2009; Clavagnier et al., 2013), thus most likely exhibiting a smaller remission rate than occlusion. Moreover, Campana et al. (2014) found that PL associated with transcranial random noise stimulation (tRNS) could improve visual acuity and contrast sensitivity substantially. Moret et al. (2018) latterly using a high-frequency transcranial random noise stimulation (hf-tRNS) combined with a short perceptual training showed that hf-tRNS could boost the transfer of PL to untrained visual functions. A strengthening of the low-level responses from the amblyopic eye might result in more balance in the mutual inhibitory interocular circuits and could indirectly result in a reduced suppressive influence. It would also be of interest to investigate the effect of the combination of these monocular interventions on the interocular suppression of amblyopes.

\section{Limitations of the Present Study}

Owing to the spatial resolution limitations of the binocular phase combination task (Wang et al., 2019), our measures of interocular suppression are limited to $1 \mathrm{c} /{ }^{\circ}$. In amblyopia, interocular suppression occurs across the entire spatial frequency range (Ding et al., 2013; Kwon et al., 2015; Zhou et al., 2018). Whether the interocular suppression is slightly greater at higher spatial frequencies (Zhou et al., 2018) is controversial because the contrast attenuation caused by the threshold deficit is often not taken into account in such measurements. It is a possibility that occlusion therapy influences interocular suppression in a spatial frequency specific manner and that our measurements of interocular suppression at $1 \mathrm{c} /{ }^{\circ}$ do not reflect and that binocular therapy and occlusion therapy differ in this respect. Even if this was the case, it would still suggest that their mode of action was different. Another proviso is that we assume that the different techniques that have been developed to measure interocular suppression are all measuring the same suppressive effects. In our study, we use a binocular phase combination task; previous measurements of the effects from binocular therapy have used a global motion approach (Black et al., 2011). A previous study (Zhou et al., 2013b) has shown that the interocular suppression measured with these different tasks while being significantly different is, however, correlated in adults with amblyopia. While we have no reason to doubt that this is not the case in children with amblyopia, we are at present assuming this. Thirdly, to make sure that patients responded to the occlusion therapy, we selected children of 9 years old or younger. Furthermore, all the subjects we studied here had anisometropic amblyopia or mixed amblyopia. We cannot therefore extend our conclusion to patients with a pure strabismic amblyopia. 


\section{DATA AVAILABILITY STATEMENT}

The datasets generated for this study are available on request to the corresponding authors.

\section{ETHICS STATEMENT}

The studies involving human participants were reviewed and approved by Ethics Committee of the Wenzhou Medical University. Written informed consent to participate in this study was provided by the participants' legal guardian/next of kin.

\section{AUTHOR CONTRIBUTIONS}

$\mathrm{HC}, \mathrm{JZ}$, and $\mathrm{RH}$ conceived the experiments. $\mathrm{YC}, \mathrm{ZH}$, and $\mathrm{YM}$ performed the experiments. $\mathrm{YC}, \mathrm{ZH}$, and JZ analyzed and interpreted the data. YC, JZ, HC, and $\mathrm{RH}$ wrote the manuscript. All authors contributed

\section{REFERENCES}

Abraham, W. C., and Bear, M. F. (1996). Metaplasticity: the plasticity of synaptic plasticity. Trends Neurosci. 19, 126-130. doi: 10.1016/s0166-2236(96)80018-x

Astle, A. T., Webb, B. S., and McGraw, P. V. (2011). The pattern of learned visual improvements in adult amblyopia. Invest. Ophthalmol. Vis. Sci. 52, 7195-7204. doi: 10.1167/iovs.11-7584

Baker, D. H., Meese, T. S., and Hess, R. F. (2008). Contrast masking in strabismic amblyopia: attenuation, noise, interocular suppression and binocular summation. Vision Res. 48, 1625-1640. doi: 10.1016/j.visres.2008.04.017

Bhola, R., Keech, R. V., Kutschke, P., Pfeifer, W., and Scott, W. E. (2006). Recurrence of amblyopia after occlusion therapy. Ophthalmology 113, 20972100. doi: 10.1016/j.ophtha.2006.04.034

Birch, E. E. (2013). Amblyopia and binocular vision. Prog. Retin. Eye Res. 33, 67-84. doi: 10.1016/j.preteyeres.2012.11.001

Birch, E. E., Li, S. L., Jost, R. M., Morale, S. E., De La Cruz, A., Stager, D. Jr., et al. (2015). Binocular iPad treatment for amblyopia in preschool children. J. AAPOS 19, 6-11. doi: 10.1016/j.jaapos.2014.09.009

Birch, E. E., Morale, S. E., Jost, R. M., De La Cruz, A., Kelly, K. R., Wang, Y. Z., et al. (2016). Assessing suppression in amblyopic children with a dichoptic eye chart. Invest. Ophthalmol. Vis. Sci. 57, 5649-5654. doi: 10.1167/iovs.16-19986

Black, J. M., Thompson, B., Maehara, G., and Hess, R. F. (2011). A compact clinical instrument for quantifying suppression. Optom. Vis. Sci. 88, 334-343. doi: 10.1097/OPX.0b013e318205a162

Bocci, T., Nasini, F., Caleo, M., Restani, L., Barloscio, D., Ardolino, G., et al. (2018). Unilateral application of cathodal tDCS reduces transcallosal inhibition and improves visual acuity in amblyopic patients. Front. Behav. Neurosci. 12:109. doi: 10.3389/fnbeh.2018.00109

Brainard, D. H. (1997). The psychophysics toolbox. Spat. Vis. 10, 433-436. doi: $10.1163 / 156856897 \times 00357$

Buzas, P., Eysel, U. T., Adorjan, P., and Kisvarday, Z. F. (2001). Axonal topography of cortical basket cells in relation to orientation, direction, and ocular dominance maps. J. Comp. Neurol. 437, 259-285. doi: 10.1002/cne. 1282

Campana, G., Camilleri, R., Moret, B., Ghin, F., and Pavan, A. (2016). Opposite effects of high- and low-frequency transcranial random noise stimulation probed with visual motion adaptation. Sci. Rep. 6:38919. doi: 10.1038/ srep38919

Campana, G., Camilleri, R., Pavan, A., Veronese, A., and Lo, G. G. (2014) Improving visual functions in adult amblyopia with combined perceptual to manuscript revision, and read and approved the submitted version.

\section{FUNDING}

This work was supported by the National Natural Science Foundation of China Grants NSFC 31970975 and 81500754, the Qianjiang Talent Project (QJD1702021), the Project of State Key Laboratory of Ophthalmology, Optometry and Visual Science, Wenzhou Medical University (K171206), and the Wenzhou Medical University grant QTJ16005 to JZ; and by the Canadian Institutes of Health Research Grant CCI-125686, NSERC grant 228103, and an ERA-NET Neuron grant (JTC2015) to RH. The sponsor or funding organization had no role in the design or conduct of this research.

\section{ACKNOWLEDGMENTS}

We thank Mr. Seung Hyun (Sam) Min for his very kind help in correcting our English.

training and transcranial random noise stimulation (tRNS): a pilot study. Front. Psychol. 5:1402. doi: 10.3389/fpsyg.2014.01402

Chen, H., He, Z., Xu, J., Mao, Y., Liang, Y., Lin, D., et al. (2019). A convenient and robust test to quantify interocular suppression for children with amblyopia. Iperception 10:2041669519864971. doi: 10.1177/2041669519864971

Chen, Z., Li, J., Liu, J., Cai, X., Yuan, J., Deng, D., et al. (2016). Monocular perceptual learning of contrast detection facilitates binocular combination in adults with anisometropic amblyopia. Sci. Rep. 6:20187. doi: 10.1038/srep2 0187

Christiansen, S. P., Lee, K. A., Morse, C. L., Repka, M. X., Sprunger, D. T., Melia, M., et al. (2018). Amblyopia preferred practice pattern (R). Ophthalmology 125, 105-142. doi: 10.1016/j.ophtha.2017.10.008

Clavagnier, S., Thompson, B., and Hess, R. F. (2013). Long lasting effects of daily theta burst rTMS sessions in the human amblyopic cortex. Brain Stimul. 6, 860-867. doi: 10.1016/j.brs.2013.04.002

de Buffon, M. (2005). Dissertation on the cause of strabismus or squint. Translated from: "dissertation sur la cause du strabismus ou des yeux louches". Mem. Acad. Roy. Sci. (Paris), 19 June 1743. Strabismus 13, 205-212. doi: 10.1080/ 09273970590934319

Ding, J., Klein, S. A., and Levi, D. M. (2013). Binocular combination in abnormal binocular vision. J. Vis. 13:14. doi: 10.1167/13.2.14

Ding, J., and Sperling, G. (2006). A gain-control theory of binocular combination. Proc. Natl. Acad. Sci. U.S.A. 103, 1141-1146. doi: 10.1073/pnas.0509629103

Ding, Z., Li, J., Spiegel, D. P., Chen, Z., Chan, L., Luo, G., et al. (2016). The effect of transcranial direct current stimulation on contrast sensitivity and visual evoked potential amplitude in adults with amblyopia. Sci. Rep. 6:19280. doi: 10.1038/srep19280

Epelbaum, M., Milleret, C., Buisseret, P., and Dufier, J. L. (1993). The sensitive period for strabismic amblyopia in humans. Ophthalmology 100, 323-327.

Feng, L., Zhou, J., Chen, L., and Hess, R. F. (2015). Sensory eye balance in surgically corrected intermittent exotropes with normal stereopsis. Sci. Rep. 5:13075. doi: 10.1038/srep13075

Frenkel, M. Y., and Bear, M. F. (2004). How monocular deprivation shifts ocular dominance in visual cortex of young mice. Neuron 44, 917-923. doi: 10.1016/j. neuron.2004.12.003

Gao, T. Y., Guo, C. X., Babu, R. J., Black, J. M., Bobier, W. R., Chakraborty, A., et al. (2018). Effectiveness of a binocular video game vs placebo video game for improving visual functions in older children, teenagers, and adults with amblyopia: a randomized clinical trial. JAMA Ophthalmol. 136, 172-181. doi: 10.1001/jamaophthalmol.2017.6090 
Hess, R. F., Mansouri, B., and Thompson, B. (2010a). A binocular approach to treating amblyopia: anti-suppression therapy. Optom. Vis. Sci. 87, 697-704. doi: 10.1097/opx.0b013e3181ea18e9

Hess, R. F., Mansouri, B., and Thompson, B. (2010b). A new binocular approach to the treatment of amblyopia in adults well beyond the critical period of visual development. Restor. Neurol. Neurosci. 28, 793-802.

Hess, R. F., Mansouri, B., and Thompson, B. (2011). Restoration of binocular vision in amblyopia. Strabismus 19, 110-118. doi: 10.3109/09273972.2011.600418

Hess, R. F., and Thompson, B. (2015). Amblyopia and the binocular approach to its therapy. Vision Res. 114, 4-16. doi: 10.1016/j.visres.2015.02.009

Holmes, J. M., Beck, R. W., Kraker, R. T., Astle, W. F., Birch, E. E., Cole, S. R., et al. (2004). Risk of amblyopia recurrence after cessation of treatment. J. AAPOS 8 , 420-428. doi: 10.1016/S1091853104001612

Holmes, J. M., Edwards, A. R., Beck, R. W., Arnold, R. W., Johnson, D. A., Klimek, D. L., et al. (2005). A randomized pilot study of near activities versus nonnear activities during patching therapy for amblyopia. J. AAPOS 9, 129-136. doi: 10.1016/j.jaapos.2004.12.014

Holmes, J. M., Kraker, R. T., Beck, R. W., Birch, E. E., Cotter, S. A., Everett, D. F., et al. (2003). A randomized trial of prescribed patching regimens for treatment of severe amblyopia in children. Ophthalmology 110, 2075-2087. doi: 10.1016/j.ophtha.2003.08.001

Holmes, J. M., Manh, V. M., Lazar, E. L., Beck, R. W., Birch, E. E., Kraker, R. T., et al. (2016). Effect of a binocular iPad game vs part-time patching in children aged 5 to 12 years with amblyopia: a randomized clinical trial. JAMA Ophthalmol. 134, 1391-1400. doi: 10.1001/jamaophthalmol.2016.4262

Huang, C. B., Zhou, J., Lu, Z. L., Feng, L., and Zhou, Y. (2009). Binocular combination in anisometropic amblyopia. J. Vis. 9, 17.1-17.16. doi: 10.1167/ 9.3.17

Huang, C. B., Zhou, Y., and Lu, Z. L. (2008). Broad bandwidth of perceptual learning in the visual system of adults with anisometropic amblyopia. Proc. Natl. Acad. Sci. U.S.A. 105, 4068-4073. doi: 10.1073/pnas.0800824105

Iny, K., Heynen, A. J., Sklar, E., and Bear, M. F. (2006). Bidirectional modifications of visual acuity induced by monocular deprivation in juvenile and adult rats. J. Neurosci. 26, 7368-7374. doi: 10.1523/JNEUROSCI.0124-06.2006

Jia, W. L., Lan, F. F., Zhao, X., Lu, Z. L., Huang, C. B., Zhao, W. X., et al. (2018). The effects of monocular training on binocular functions in anisometropic amblyopia. Vision Res. 152, 74-83. doi: 10.1016/j.visres.2017.02.008

Kehrein, S., Kohnen, T., and Fronius, M. (2016). Dynamics of interocular suppression in amblyopic children during electronically monitored occlusion therapy: first insight. Strabismus 24, 51-62. doi: 10.3109/09273972.2016. 1170047

Kelly, K. R., Jost, R. M., Dao, L., Beauchamp, C. L., Leffler, J. N., and Birch, E. E. (2016). Binocular iPad game vs patching for treatment of amblyopia in children: a randomized clinical trial. JAMA Ophthalmol. 134, 1402-1408. doi: 10.1001/jamaophthalmol.2016.4224

Kelly, K. R., Jost, R. M., Wang, Y. Z., Dao, L., Beauchamp, C. L., Leffler, J. N., et al. (2018). Improved binocular outcomes following binocular treatment for childhood amblyopia. Invest. Ophthalmol. Vis. Sci. 59, 1221-1228. doi: 10.1167/ iovs.17-23235

Knox, P. J., Simmers, A. J., Gray, L. S., and Cleary, M. (2012). An exploratory study: prolonged periods of binocular stimulation can provide an effective treatment for childhood amblyopia. Invest. Ophthalmol. Vis. Sci. 53, 817-824. doi: $10.1167 /$ iovs.11-8219

Kwon, M., Lu, Z. L., Miller, A., Kazlas, M., Hunter, D. G., and Bex, P. J. (2014). Assessing binocular interaction in amblyopia and its clinical feasibility. PLoS One 9:e100156. doi: 10.1371/journal.pone.0100156

Kwon, M., Wiecek, E., Dakin, S. C., and Bex, P. J. (2015). Spatial-frequency dependent binocular imbalance in amblyopia. Sci. Rep. 5:17181. doi: 10.1038/ srep17181

Levi, D. M. (2005). Perceptual learning in adults with amblyopia: a reevaluation of critical periods in human vision. Dev. Psychobiol. 46, 222-232. doi: 10.1002/dev. 20050

Levi, D. M., and Li, R. W. (2009). Perceptual learning as a potential treatment for amblyopia: a mini-review. Vision Res. 49, 2535-2549. doi: 10.1016/j.visres.2009. 02.010

Levi, D. M., Polat, U., and Hu, Y. S. (1997). Improvement in Vernier acuity in adults with amblyopia. Practice makes better. Invest. Ophthalmol. Vis. Sci. 38, 1493-1510. doi: 10.1007/BF00947068
Li, J., Thompson, B., Deng, D., Chan, L. Y., Yu, M., and Hess, R. F. (2013). Dichoptic training enables the adult amblyopic brain to learn. Curr. Biol. 23, R308-R309. doi: 10.1016/j.cub.2013.01.059

Li, J., Thompson, B., Lam, C. S., Deng, D., Chan, L. Y., Maehara, G., et al. (2011). The role of suppression in amblyopia. Invest. Ophthalmol. Vis. Sci. 52, 4169-4176. doi: 10.1167/iovs.11-7233

Li, R. W., and Levi, D. M. (2004). Characterizing the mechanisms of improvement for position discrimination in adult amblyopia. J. Vis. 4, 476-487. doi: 10.1167/ 4.6.7

Li, S. L., Jost, R. M., Morale, S. E., De La Cruz, A., Dao, L., Stager, D. Jr., et al. (2015). Binocular iPad treatment of amblyopia for lasting improvement of visual acuity. JAMA Ophthalmol. 133, 479-480. doi: 10.1001/jamaophthalmol.2014. 5515

Li, S. L., Jost, R. M., Morale, S. E., Stager, D. R., Dao, L., Stager, D., et al. (2014). A binocular iPad treatment for amblyopic children. Eye 28, 1246-1253. doi: 10.1038/eye.2014.165

Manh, V. M., Holmes, J. M., Lazar, E. L., Kraker, R. T., Wallace, D. K., Kulp, M. T., et al. (2018). A randomized trial of a binocular iPad game versus part-time patching in children aged 13 to 16 years with amblyopia. Am. J. Ophthalmol. 186, 104-115. doi: 10.1016/j.ajo.2017.11.017

Mansouri, B., Singh, P., Globa, A., and Pearson, P. (2014). Binocular training reduces amblyopic visual acuity impairment. Strabismus 22, 1-6. doi: 10.3109/ 09273972.2013.877945

Mansouri, B., Thompson, B., and Hess, R. F. (2008). Measurement of suprathreshold binocular interactions in amblyopia. Vision Res. 48, 2775-2784. doi: 10.1016/j.visres.2008.09.002

Min, S. H., Baldwin, A. S., and Hess, R. F. (2019). Ocular dominance plasticity: a binocular combination task finds no cumulative effect with repeated patching. Vision Res. 161, 36-42. doi: 10.1016/j.visres.2019.05.007

Moret, B., Camilleri, R., Pavan, A., Lo Giudice, G., Veronese, A., Rizzo, R., et al. (2018). Differential effects of high-frequency transcranial random noise stimulation (hf-tRNS) on contrast sensitivity and visual acuity when combined with a short perceptual training in adults with amblyopia. Neuropsychologia 114, 125-133. doi: 10.1016/j.neuropsychologia.2018.04.017

Moseley, M. J., Fielder, A. R., Irwin, M., Jones, H. S., and Auld, R. J. (1997). Effectiveness of occlusion therapy in ametropic amblyopia: a pilot study. $\mathrm{Br}$. J. Ophthalmol. 81, 956-961. doi: 10.1136/bjo.81.11.956

Mou, T. (1966). Logarithmic visual acuity chart and five-score recording. Chin. J. Ophthalmol. 13, 96-106.

Nitsche, M. A., and Paulus, W. (2000). Excitability changes induced in the human motor cortex by weak transcranial direct current stimulation. J. Physiol. 527, 633-639. doi: 10.1111/j.1469-7793.2000.t01-1-00633.x

Pediatric Eye Disease Investigator Group Writing Committee Rutstein, R. P., Quinn, G. E., Lazar, E. L., Beck, R. W., Bonsall, D. J., et al. (2010). A randomized trial comparing Bangerter filters and patching for the treatment of moderate amblyopia in children. Ophthalmology 117, 998-1004.e6.

Pelli, D. G. (1997). The VideoToolbox software for visual psychophysics: transforming numbers into movies. Spat. Vis. 10, 437-442. doi: 10.1163/ $156856897 \times 00366$

Piano, M., and Newsham, D. (2015). A pilot study examining density of suppression measurement in strabismus. Strabismus 23, 14-21. doi: 10.3109/ 09273972.2014.1002621

Pineles, S. L., Aakalu, V. K., Hutchinson, A. K., Galvin, J. A., Heidary, G., Binenbaum, G., et al. (2019). Binocular treatment of amblyopia: a report by the American academy of ophthalmology. Ophthalmology doi: 10.1016/j.ophtha. 2019.08.024 [Epub ahead of print].

Polat, U., Ma-Naim, T., Belkin, M., and Sagi, D. (2004). Improving vision in adult amblyopia by perceptual learning. Proc. Natl. Acad. Sci. U.S.A. 101, 6692-6697. doi: 10.1073/pnas.0401200101

Ren, Z., Zhou, J., Yao, Z., Wang, Z., Yuan, N., Xu, G., et al. (2016). Neuronal basis of perceptual learning in striate cortex. Sci. Rep. 6:24769. doi: 10.1038/srep 24769

Repka, M. X., Beck, R. W., Holmes, J. M., Birch, E. E., Chandler, D. L., Cotter, S. A., et al. (2003). A randomized trial of patching regimens for treatment of moderate amblyopia in children. Arch. Ophthalmol. 121, 603-611. doi: 10.1001/archopht. 121.5.603

Sengpiel, F., Blakemore, C., Kind, P. C., and Harrad, R. (1994). Interocular suppression in the visual cortex of strabismic cats. J. Neurosci. 14, 6855-6871. 
Sengpiel, F., Jirmann, K. U., Vorobyov, V., and Eysel, U. T. (2006). Strabismic suppression is mediated by inhibitory interactions in the primary visual cortex. Cereb. Cortex 16, 1750-1758. doi: 10.1093/cercor/bhj110

Sheynin, Y., Chamoun, M., Baldwin, A. S., Rosa-Neto, P., Hess, R. F., and Vaucher, E. (2019). Cholinergic potentiation alters perceptual eye dominance plasticity induced by a few hours of monocular patching in adults. Front. Neurosci. 13:22. doi: 10.3389/fnins.2019.00022

Spiegel, D. P., Li, J., Hess, R. F., Byblow, W. D., Deng, D., Yu, M., et al. (2013). Transcranial direct current stimulation enhances recovery of stereopsis in adults with amblyopia. Neurotherapeutics 10, 831-839. doi: 10.1007/s13311013-0200-y

Stewart, C. E., Moseley, M. J., Stephens, D. A., and Fielder, A. R. (2004). Treatment dose-response in amblyopia therapy: the Monitored Occlusion Treatment of Amblyopia Study (MOTAS). Invest. Ophthalmol. Vis. Sci. 45, 3048-3054.

Stewart, C. E., Stephens, D. A., Fielder, A. R., Moseley, M. J., and Cooperative, M. (2007). Modeling dose-response in amblyopia: toward a child-specific treatment plan. Invest. Ophthalmol. Vis. Sci. 48, 2589-2594.

Thompson, B., Mansouri, B., Koski, L., and Hess, R. F. (2008). Brain plasticity in the adult: modulation of function in amblyopia with rTMS. Curr. Biol. 18, 1067-1071. doi: 10.1016/j.cub.2008.06.052

To, L., Thompson, B., Blum, J. R., Maehara, G., Hess, R. F., and Cooperstock, J. R. (2011). A game platform for treatment of amblyopia. IEEE Trans. Neural Syst. Rehabil. Eng. 19, 280-289. doi: 10.1109/TNSRE.2011.2115255

Wang, J., Feng, L., Wang, Y., Zhou, J., and Hess, R. F. (2018). Binocular benefits of optical treatment in anisometropic amblyopia. J. Vis. 18:6. doi: 10.1167/18.4.6

Wang, J., Neely, D. E., Galli, J., Schliesser, J., Graves, A., Damarjian, T. G., et al. (2016). A pilot randomized clinical trial of intermittent occlusion therapy liquid crystal glasses versus traditional patching for treatment of moderate unilateral amblyopia. J. AAPOS 20, 326-331. doi: 10.1016/j.jaapos.2016.05.014

Wang, Y., Cui, L., He, Z., Lin, W., Qu, J., Lu, F., et al. (2018). On the relationship between sensory eye dominance and stereopsis in the normal-sighted adult population: normative data. Front. Hum. Neurosci. 12:357. doi: 10.3389/fnhum. 2018.00357

Wang, Y., He, Z., Liang, Y., Chen, Y., Gong, L., Mao, Y., et al. (2019). The binocular balance at high spatial frequencies as revealed by the binocular orientation combination task. Front. Hum. Neurosci. 13:106. doi: 10.3389/fnhum.2019. 00106

Webber, A. L., Wood, J. M., and Thompson, B. (2016). Fine motor skills of children with amblyopia improve following binocular treatment. Invest. Ophthalmol. Vis. Sci. 57, 4713-4720. doi: 10.1167/iovs.16-19797

Yu, Q., Zhang, P., Qiu, J., and Fang, F. (2016). Perceptual learning of contrast detection in the human lateral geniculate nucleus. Curr. Biol. 26, 3176-3182. doi: $10.1016 /$ j.cub.2016.09.034

Zhou, J., Clavagnier, S., and Hess, R. F. (2013a). Short-term monocular deprivation strengthens the patched eye's contribution to binocular combination. J. Vis. 13:12. doi: $10.1167 / 13.5 .12$
Zhou, J., Huang, P. C., and Hess, R. F. (2013b). Interocular suppression in amblyopia for global orientation processing. J. Vis. 13:19. doi: 10.1167/ 13.5.19

Zhou, J., Jia, W., Huang, C. B., and Hess, R. F. (2013c). The effect of unilateral mean luminance on binocular combination in normal and amblyopic vision. Sci. Rep. 3:2012. doi: 10.1038/srep02012

Zhou, J., He, Z., Wu, Y., Chen, Y., Chen, X., Liang, Y., et al. (2019). Inverse occlusion: a binocularly motivated treatment for amblyopia. Neural Plast. 2019:5157628. doi: 10.1155/2019/5157628

Zhou, J., Liu, R., Zhou, Y., and Hess, R. F. (2014a). Binocular combination of second-order stimuli. PLoS One 9:e84632. doi: 10.1371/journal.pone.0084632

Zhou, J., Reynaud, A., and Hess, R. F. (2014b). Real-time modulation of perceptual eye dominance in humans. Proc. Biol. Sci. 281:20141717. doi: 10.1098/rspb. 2014.1717

Zhou, J., Reynaud, A., and Hess, R. F. (2017a). Aerobic exercise effects on ocular dominance plasticity with a phase combination task in human adults. Neural Plast. 2017:4780876. doi: 10.1155/2017/4780876

Zhou, J., Reynaud, A., Kim, Y. J., Mullen, K. T., and Hess, R. F. (2017b). Chromatic and achromatic monocular deprivation produce separable changes of eye dominance in adults. Proc. Biol. Sci. 284:20171669. doi: 10.1098/rspb.2017. 1669

Zhou, J., Wang, Y., Feng, L., Wang, J., and Hess, R. F. (2017c). Straightening the eyes doesn't rebalance the brain. Front. Hum. Neurosci. 11:453. doi: 10.3389/ fnhum.2017.00453

Zhou, J., Reynaud, A., Yao, Z., Liu, R., Feng, L., Zhou, Y., et al. (2018). Amblyopic suppression: passive attenuation, enhanced dichoptic masking by the fellow eye or reduced dichoptic masking by the amblyopic eye? Invest. Ophthalmol. Vis. Sci. 59, 4190-4197. doi: 10.1167/iovs.18-24206

Zhou, J., Zhang, Y., Dai, Y., Zhao, H., Liu, R., Hou, F., et al. (2012). The eye limits the brain's learning potential. Sci. Rep. 2:364. doi: 10.1038/srep0 0364

Zhou, Y., Huang, C., Xu, P., Tao, L., Qiu, Z., Li, X., et al. (2006). Perceptual learning improves contrast sensitivity and visual acuity in adults with anisometropic amblyopia. Vision Res. 46, 739-750. doi: 10.1016/j.visres.2005. 07.031

Conflict of Interest: The authors declare that the research was conducted in the absence of any commercial or financial relationships that could be construed as a potential conflict of interest.

Copyright (C) 2020 Chen, He, Mao, Chen, Zhou and Hess. This is an open-access article distributed under the terms of the Creative Commons Attribution License (CC BY). The use, distribution or reproduction in other forums is permitted, provided the original author(s) and the copyright owner(s) are credited and that the original publication in this journal is cited, in accordance with accepted academic practice. No use, distribution or reproduction is permitted which does not comply with these terms. 